\title{
Editorial
}

\section{Difusión de la Ciencia Gerencial}

Estamos sumergidos en una dinámica de múltiples caras, que muchas veces nos obliga a aproximarnos a la realidad en forma equivocada. No hay determinismo que prevalezca en la forma como vemos las cosas. Sólo creemos que debemos seguir buscando lo que es la realidad. Y en la büsqueda por entender la realidad, se ha desarrollado ciencia. Pero, ciencia no es cualquier cosa. Ciencia es básicamente investigación. Hemos pasado de un conocimiento enciclopédico a uno mucho más estrecho derivado de la especialización. Sin embargo, tal especialización muchas veces hace del hombre un ser minusválido de cultura integral, que ni siquiera es poseedor de una parte de la totalidad del conocimiento en el cual se ha especializado.

En estos dias, se habla de ciencia, tanto como se habla de gerencia. Se ha pretendido llamar ciencia a un conocimiento fragmentado derivado de la especialización. Para Karl Popper los grandes progresos sólo tienen lugar cuando confluyen las disciplinas. Y es que no existen dos ciencias, sino una sola. Ciencia y gerencia se vinculan en un proceso de búsqueda de mecanismos para tomar mejores decisiones. Y en ese proceso por desarrollar temas sobre ciencias gerenciales, las personas se interesan por entender los fundamentos teóricos que la sustentan, a medida que utilizan las técnicas que facilitan la toma de decisiones.

La Gerencia Científica atrae, cada vez más, a personas que se interesan por esta área del conocimiento. La presión que ejercen las industrias y las organizaciones en general por entender los temas gerenciales, promueve su análisis y hace que las universidades e institutos especializados provean más educación práctica sobre esos tópicos. Sin embargo, en ocasiones se aborda la gerencia en forma pragmática, aumentando las dudas sobre la naturaleza cientifica de los estudios gerenciales. El mercado de la literatura sobre esta disciplina está plagado de una oferta de fórmulas salvadoras para desarrollar gerencia. Ni siquiera las nuevas tecnologias de información pueden proveemos de una capacidad sobrehumana para asimilar el cúmulo de información disponible. Se debe ser selectivo en lo que leemos y procesamos. La velocidad con que la información cambia nos obliga a discriminar lo que puede ser usado para generar conocimiento. Afortunadamente, el camino de la gerencia cuenta con investigadores, que nos permiten confiar que el estudio de la gerencia puede ser realizado con rigor científico.

La Revista Venezolana de Gerencia está dedicada al análisis de temas críticos que indudablemente reafirman que el estudio de la gerencia corresponde a una disciplina científica. Los procedimientos que se realizan para la selección de articulos son consistentes en el mantenimiento de altos y racionales estándares internacionales. El proceso de revisión y aceptación de articulos está sometido al criterio de árbitros con amplios conocimientos de los temas tratados, 
quienes deciden sobre lo significante que pudiera ser para la literatura cientifica la publicación de tales trabajos. Finalmente, un comité de editores evalúa la calidad y originalidad de los trabajos para decidir la pertinencia que pueda tener para los lectores. La Revista Venezolana de Gerencia cumple un rol fundamental dentro de la difusión cientifica; es por ello que la revista cuenta además con un selecto grupo de asesores quienes se dedican a su difusión, a la vez que aportan sus experiencias a fin de elevar la calidad de trabajos para que el público interesado pueda entenderios y evaluarios.

Con este número doce de la Revista Venezolana de Gerencia se pone a disposición del lector trabajos de elevada calidad científica de reconocidos autores, tanto nacionales como internacionales. Ellos son: Bernardo KIiksberg quien es coordinador del Instituto Interamerica-. no para el Desarrollo Social (INDES/BID) y Protesor Honorario de la Universidad Nacional de Buenos Aires, Argentina; Ignacio Pérez Salgado, director de la Maestría en Gerencia Pública de la Universidad de Santiago de Chile; Marcela Fernández, investigadora del Programa ALDEA XXI. Universidad Nacional de Quilmes; Rosa Salom, Licenciada en Comunicación Social, Master en Radio \& Televisión, Master in European Business Comunicación y Coordinadora del Área de Nuevas Tecnologias del Centro de Investigaciones de la Comunicación y la Información de La Universidad del Zulia; Mercedes Ferrer, Arquitecto, Master en Planificación Urbana y Regional en la Universidad de Newcastle, Inglaterra e Investigadora de la Universidad del Zulia; Mercy Narváez, Licenciada en Administración, Magister en Gerencia de Empresas; Gladys Fernández, Licenciada en Contaduria Püblica y Master en Educación; Nerio Vilchez, pedagogo y Master en Educación; y Arelis Abreu, Magister en Gerencia Educativa.

Estoy seguro que con la publicación de este número de la Revista Venezolana de Gerencia se incrementará el acervo científico de los tópicos interesantes que aqui aparecen.

Jesús Alberto Andrade*

* Asesor de la Revista Venezolana de Gerencia. Investigador sobre tópicos de Gerencia de la Información. Economista. Magister en Computación Aplicada y Master en Gerencia de los Sistemas de Información. 